\title{
Coordinated temporal and spatial control of motor neuron and serotonergic neuron generation from a common pool of CNS progenitors
}

\author{
Alexandre Pattyn, ${ }^{1,2}$ Anna Vallstedt, ${ }^{1}$ José M. Dias, ${ }^{1}$ Omar Abdel Samad, ${ }^{3}$ Robb Krumlauf, ${ }^{4}$ \\ Filippo M. Rijli, ${ }^{3}$ Jean-Francois Brunet, ${ }^{2}$ and Johan Ericson ${ }^{1,5}$ \\ ${ }^{1}$ Department of Cell and Molecular Biology, Karolinska Institute S-171 77 Stockholm, Sweden; ${ }^{2}$ CNRS UMR8542 Ecole \\ Normale Supérieure, Département de Biologie 75005 Paris, France; ${ }^{3}$ Institut de Génétique et de Biologie Moléculaire et \\ Cellulaire CNRS/INSERM/ULP, Collège de France BP 163-67404 Illkirch Cedex, CU de Strasbourg, France; ${ }^{4}$ Stowers \\ Institute, Kansas City, Missouri 64110, USA
}

Neural progenitor cells often produce distinct types of neurons in a specific order, but the determinants that control the sequential generation of distinct neuronal subclasses in the vertebrate CNS remain poorly defined. We examined the sequential generation of visceral motor neurons and serotonergic neurons from a common pool of neural progenitors located in the ventral hindbrain. We found that the temporal specification of these neurons varies along the anterior-posterior axis of the hindbrain, and that the timing of their generation critically depends on the integrated activities of Nkx- and Hox-class homeodomain proteins. A primary function of these proteins is to coordinate the spatial and temporal activation of the homeodomain protein Phox $2 b$, which in turn acts as a binary switch in the selection of motor neuron or serotonergic neuronal fate. These findings assign new roles for Nkx, Hox, and Phox2 proteins in the control of temporal neuronal fate determination, and link spatial and temporal patterning of CNS neuronal fates.

[Keywords: CNS; development; motor neuron; 5HT; patterning; homeodomain]

Received November 26, 2002; revised version accepted January 10, 2003.

Neuronal cell diversity is established by mechanisms that operate in space and over time during central nervous system (CNS) development. Insight has been obtained regarding the initial steps of spatial patterning of neurons along the dorsal-ventral (DV) and anterior-posterior (AP) axes of the neural tube (Lumsden and Krumlauf 1996; Jessell 2000). Local inductive signals determine the spatial pattern of expression of transcription factors along both these axes, so that neural progenitors at different positions acquire distinct molecular identities. In the ventral neural tube, neuronal fate along the DV axis depends on the Shh-mediated patterning of Nkx-, Dbx-, Pax-, and Irx-class homeodomain (HD) proteins (Briscoe et al. 2000). Along the AP axis, the overlapping, or nested, expression pattern of Hox HD proteins provides positional values that influence the fate of neurons (Lumsden and Krumlauf 1996). Despite significant advances, however, DV and AP patterning have generally been analyzed independently, leaving open the is-

${ }^{5}$ Corresponding author.

E-MAIL Johan.Ericson@cmb.ki.se; FAX 46-8-30-83-74.

Article published online ahead of print. Article and publication date are at http://www.genesdev.org/cgi/doi/10.1101/gad.255803. sue as to what degree these orthogonal patterning mechanisms are integrated (Davenne et al. 1999; Gaufo et al. 2000). Compared to spatial patterning, little is known about the mechanisms that underlie how neural progenitors produce distinct types of neurons in a specific temporal order. Studies of the retina (Livesey and Cepko 2001) and developing neo-cortex (Monuki and Walsh 2001) suggest that the sequential production of different neuronal subtypes reflects temporal changes in neural progenitors, either in response to extrinsic cues or mechanisms intrinsic to neural progenitor cells. Recent data indicate that modulation of Notch signaling by the bHLH protein Mash1 and the HD proteins Dlx1/2 may control the sequential specification of progenitors in subcortical areas of the telencephalon (Yun et al. 2002). Apart from this, few molecular determinants that influence these temporal processes in the vertebrate CNS have been identified to date.

\section{Results}

To address how spatial and temporal aspects of cell patterning are integrated during development, we examined the sequential generation of visceral motor neurons 
(vMNs) and serotonergic (S) neurons from Nkx2.2 $2^{+}$progenitors (Briscoe et al. 1999) in the ventral hindbrain. S neurons are initially detected as two distinct cell groups, one rostral and one caudal (Lidov and Molliver 1982; Aitken and Tork 1988), indicating that the generation of these neurons is interrupted along the AP axis of the hindbrain. We localized the gap between these two groups of $S$ neurons to rhombomere $(r) 4$, by mapping the exclusion of pet1 expression, an early marker for S neurons (Hendricks et al. 1999), to the r4-specific expression of Hoxb1 (Fig. 1a,b; Studer et al. 1996). S neurons were excluded from $\mathrm{r} 4$, whereas they could be detected in a position ventral to vMNs at all other levels of the hindbrain at embryonic day 11.5 (E11.5; Fig. 1c,d). BrdU birthdating analyses revealed that in $\mathrm{r} 4$, vMNs are produced at a high rate between E9.5 and E11.5, whereas at other axial levels most vMNs have been generated prior to E10.5 (Fig. 1e; see also Fig. 2a). These data reveal that the exclusion of $\mathrm{S}$ neurons from $\mathrm{r} 4$ is accompanied by a prolonged generation of vMNs (Fig. 1v).

The generation of vMNs precedes that of $S$ neurons (Taber-Pierce 1973; Briscoe et al. 1999), and we next examined the precise spatial and temporal generation of these neuronal subtypes in relation to $\mathrm{Nkx} 2.2^{+}$progenitors. Phox $2 b$, an HD protein required for the generation of hindbrain vMNs (Pattyn et al. 2000), is expressed in Nkx2.2 $2^{+}$vMN progenitors and in postmitotic vMNs that also express Isl1 (Ericson et al. 1997). At early stages (E9-E9.5), numerous vMNs but no $\mathrm{S}$ neurons are produced (Fig. 1f), and Phox2b expression was detected in most Nkx2.2 $2^{+}$progenitors, independent of axial level (Fig. 1p). At this stage, essentially all Nkx2.2+ progenitors expressed the HD proteins Nkx6.1 (Sander et al. 2000), Nkx6.2 (Vallstedt et al. 2001), and Nkx2.9 (Fig. $1 \mathrm{~g}, \mathrm{j}, \mathrm{m}$; Briscoe et al. 1999). These data show that Nkx $2.2^{+}$progenitors initially represent a largely uniform progenitor population, and that all or most cells are devoted to produce vMNs. Subsequently, the expression of Phox $2 \mathrm{~b}$ and Nkx2.9 became, within the Nkx2.2+ domain, dorsally restricted at all axial levels except $\mathrm{r} 4$ (see below). At E10.5, only the dorsal half of the $\mathrm{Nkx} 2.2^{+}$ domain expressed Phox $2 \mathrm{~b}$ and Nkx2.9 (Fig. 1h,n,q), and this dorsal restriction correlated with a cessation of vMN production and the initiation of S-neuron generation (Fig. 1f). This observation suggests that only the dorsal Nkx2.2 $2^{+} / \mathrm{Nkx} 2.9^{+} /$Phox $2 \mathrm{~b}^{+}$subpopulation continues to produce motor neurons at E10.5 and raises the possibility that $S$ neurons, in turn, derive from ventral $\mathrm{Nkx} 2.2^{+}$progenitors that have ceased to express Phox $2 \mathrm{~b}$ and $\mathrm{Nkx2.9}$. In support for this, the initial expression of pet1 at E10.75 was detected in a position immediately ventral to Phox $2 \mathrm{~b}^{+} / \mathrm{Is} 11^{+}$motor neurons and dorsal to the $\mathrm{Shh}^{+}$floor plate (Fig. 1t). Because the first S neurons to be generated have completed their final round of DNA synthesis by E10.5 (Fig. 1f) and newly-born neurons initially migrate in a strict medial-to-lateral fashion (Leber and Sanes 1995), these data strongly suggest that S neurons derive from ventral $\mathrm{Nkx} 2.2^{+} / \mathrm{Nkx} 2.9^{-} / \mathrm{Phox}_{2} \mathrm{~b}^{-}$progenitors that by E10.5 no longer produce vMNs (Fig. 1v). Moreover, although Nkx2.9 became restricted to dorsal
$\mathrm{Nkx} 2.2^{+}$progenitors in $\mathrm{r} 4$, the progenitor expression of Phox $2 b$ continued to span the entire width of the Nkx2.2 ${ }^{+}$domain up to E11.5 at this level (Fig. 1i,o,r; data not shown). These data show that the exclusion of $S$ neurons and the extended phase of vMN production observed in $\mathrm{r} 4$ correlate with an extended temporal and spatial progenitor expression of Phox2b (Fig. 1v).

What factors control the sequential generation of vMNs and S neurons in the hindbrain? Previous studies showed that Nkx6.1 and Nkx6.2 have a central role in DV patterning and in the specification of somatic MNs, which are generated in a position immediately dorsal to vMNs (Sander et al. 2000; Vallstedt et al. 2001). Nkx6.1 and Nkx6.2 are coexpressed in all Nkx2.2+ progenitors in the hindbrain (Fig. 1g-1), and we therefore investigated whether these HD proteins also influence the generation of vMNs and $S$ neurons. Because these proteins have overlapping functions (Vallstedt et al. 2001), we focused our analysis on $N k \times 6.1$ and $N k x 6.2$ compound mutant mice (Nkx6 mutants). The number of Nkx2.2+/Phox $2 \mathrm{~b}^{+}$ vMN progenitors and Is $11^{+} / \mathrm{Phox}_{2} \mathrm{~b}^{+}$neurons was similar in Nkx6 mutants and control embryos at most hindbrain levels between E9 and E10.5 (Fig. 2a). Thus, in contrast to somatic MNs (Vallstedt et al. 2001), Nkx6 proteins are dispensable for the initial specification of vMN fate. We noticed, however, that the number of Nkx2.2 $/ \mathrm{Phox}^{+} \mathrm{b}^{+}$ vMN progenitors, and the total number of vMNs generated, were drastically reduced at $\mathrm{r} 4$ levels in Nkx6 mutants (Fig. 2h-m). Quantification of Phox2b expression in $\mathrm{Nkx} 2.2^{+}$progenitors over time indicated that vMN generation in $\mathrm{r} 4$ was prematurely arrested at approximately E10.5, and the remaining expression of Phox $2 \mathrm{~b}$ was largely confined to dorsal $\mathrm{Nkx} 2.2^{+} / \mathrm{Nkx} 2.9^{+}$progenitors (Fig. 2a-i). These data suggested that $\mathrm{r} 4$-progenitors in Nkx6 mutants adopt a profile of vMN generation similar to that of other hindbrain levels, and that the loss of Nkx6 function primarily affects the late phase of vMN generation unique to 4 (Fig. 1v). Strikingly, the reduced production of vMNs in Nkx6 mutants in $\mathrm{r} 4$ was accompanied by ectopic generation of $S$ neurons, as indicated by a continuous expression of pet1 along the AP axis of the hindbrain and the detection of $S$ neurons ventral to vMNs in $\mathrm{r} 4$ at E11.5 (Fig. $2 \mathrm{j}-\mathrm{q}$ ).

The selective requirement for Nkx6 proteins to promote vMN and suppress $S$ neuron generation in $\mathrm{r} 4$ uncovers an unanticipated role for these HD proteins in AP patterning. Because Nkx6.1 and Nkx6.2 are coexpressed by all $\mathrm{Nkx} 2.2^{+}$progenitors in the hindbrain (Fig. 1g-1), we reasoned that the AP-specific mode of action of these proteins must be indirect. We therefore examined the expression of Hox genes implicated in the establishment of $r 4$ identity of the hindbrain, and found that the expression of Hoxb1 was extinguished in the ventral half of r4 at E11.5 in Nkx6 mutants (Fig. 2r,s). Several other Hox genes appeared unaffected, indicating that the overall AP identity of the hindbrain is not perturbed in these mice (Fig. 2t, $\mathrm{u}$; data not shown). Analysis of Hoxb1 expression in Nkx6 mutants at earlier stages revealed a normal expression pattern at E9.5, and a reduction of Hoxb1 expression levels was first detected at E10.5 (Fig. 2v-y). 

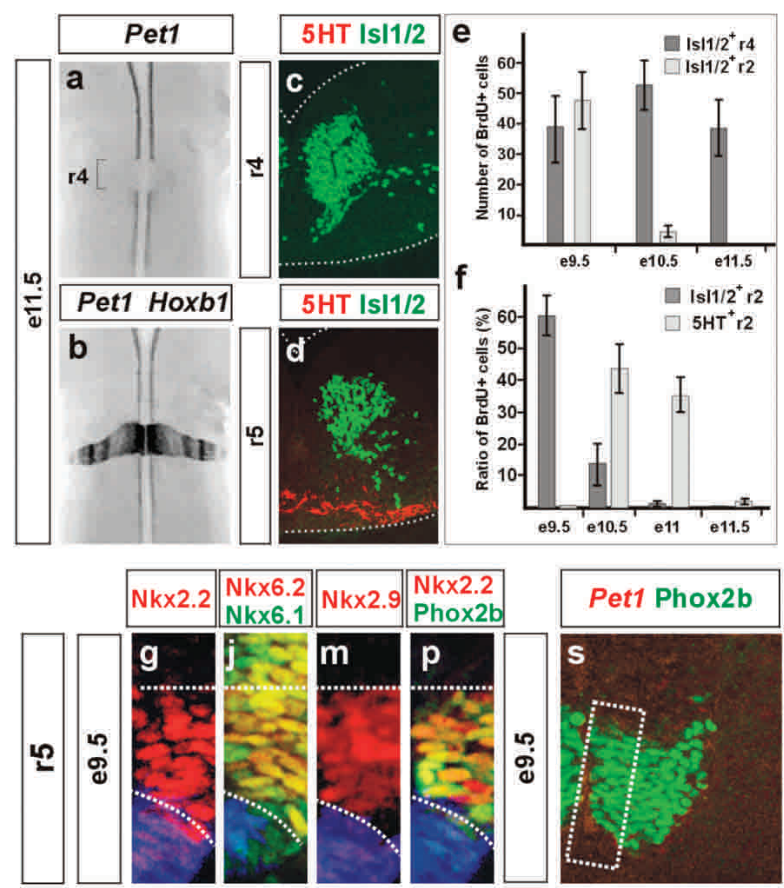

Nkx2.2
Pho $2 \mathrm{~b}$
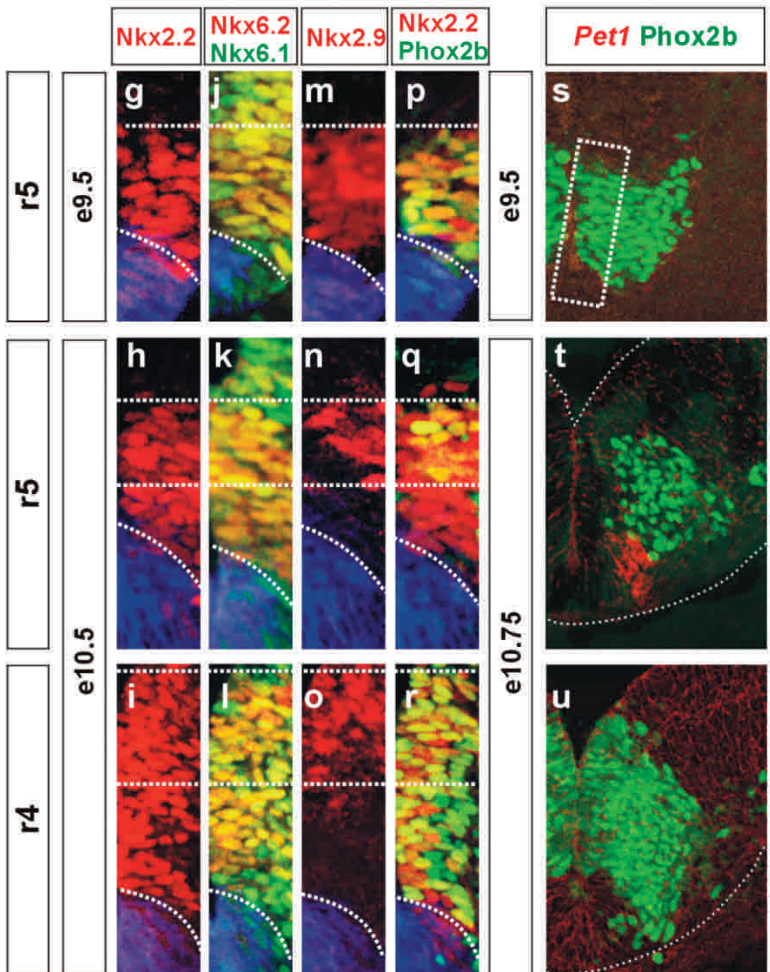

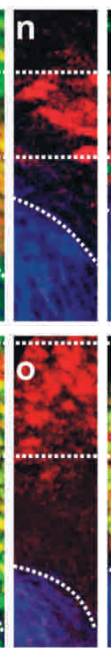

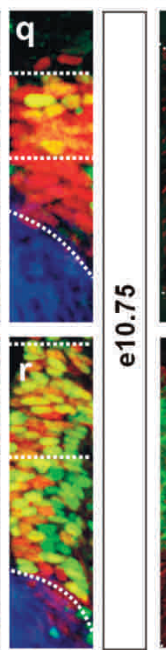
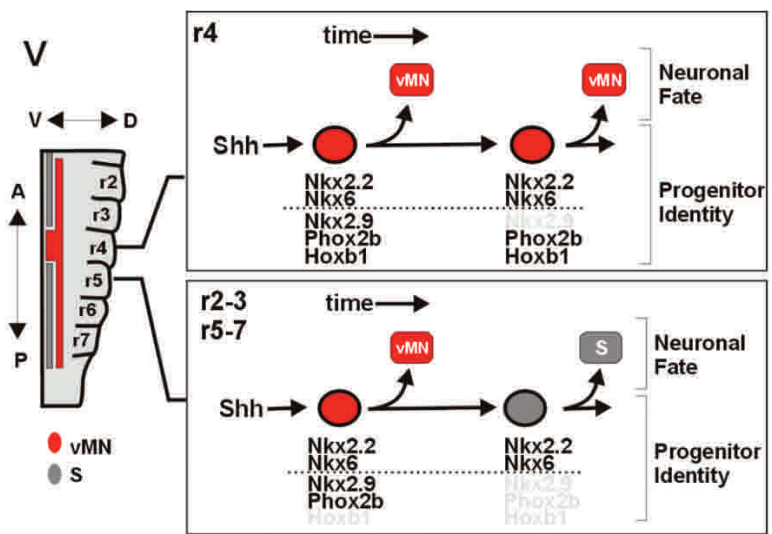

Figure 1. Spatial and temporal profile of visceral motor-neuron and S-neuron generation in the hindbrain. $(a, b)$ Micrographs show dorsal view of flat-mounted hindbrain. S neurons are generated at all axial levels of the hindbrain except in r4, as indicated by whole-mount analysis of pet 1 expression $(a)$ and the r4-specific expression of Hoxb1 $(b)$ at E11.5. $(c, d) \mathrm{S}$ neurons, as detected by the expression of serotonin $(5 \mathrm{HT})$, are present ventral to Isl1 ${ }^{+}$vMNs in $\mathrm{r} 5(d)$ but not in $\mathrm{r} 4(c) \cdot(e, f)$ BrdU birthdate analysis of vMNs and $\mathrm{S}$ neurons. vMNs in $\mathrm{r} 4$ are produced up to E11.5, whereas most r2-derived vMNs have been generated by E10.5 (e). The generation of vMNs precedes the generation of $\mathrm{S}$ neurons $(f)$. Numbers in $e$ and $f$ derive from counting three sections at the r2 or r4 level per embryo. Four embryos per stage were examined, mean \pm S.D. Consideration was taken in $e$ that r4-derived facial branchial motor neurons migrate caudally. $(g-u)$ Transverse sections through $\mathrm{r} 5$ and $\mathrm{r} 4$ levels of the hindbrain. In $\mathrm{r} 5$ at E9.5, most Nkx2.2+ progenitors $(g)$ express Nkx2.9 $(m)$ and Phox2b $(p)$. In r5 at E10.5, the expression of Nkx2.9 $(n)$ and Phox $2 \mathrm{~b}(q)$ has become restricted to the dorsal half of the $\mathrm{Nkx2} .2^{+}$domain $(h)$. A similar restriction of $\mathrm{Nkx} 2.9$ and Phox $2 \mathrm{~b}$ expression was detected at all axial levels (data not shown) except in $\mathrm{r} 4$. In $\mathrm{r} 4$ at E10.5, Nkx2.9 (o) is dorsally restricted but Phox2b expression $(r)$ continues to span the entire $\mathrm{Nkx} 2.2^{+}$domain (i). Nkx6.1 and Nkx6.2 are coexpressed in all hindbrain $\mathrm{Nkx} 2.2^{+}$progenitors independent of axial level over this period $(j-1)$. The vMN marker Phox 2b, but not the S-cell marker pet1, is detected in r5 at E9.5 (s). Dashed box in $s$ indicates progenitor domain shown in $g-u$. In $\mathrm{r} 5$ at E10.75, pet1 expression is detected ventral to Phox $2 \mathrm{~b}^{+}$cells $(t)$. At $\mathrm{r} 4$ levels at E10.75, extensive expression of Phox $2 b$ but no expression of pet 1 could be detected $(u)$. (v) Summary of the spatial and temporal generation of vMNs and S neurons in the hindbrain.
Thus, Nkx6 proteins are dispensable for the initial phase of ventral Hoxb1 expression, but are necessary to maintain high levels of Hoxb1 expression in the ventral half of $\mathrm{r} 4$ from E10.5.

AP positional values in the hindbrain are established soon after neural tube closure (Guthrie et al. 1992; Simon et al. 1995), and the role of Hoxb1 in conferring r4 identity is well documented (Studer et al. 1996; Bell et al. 1999; Gaufo et al. 2000). The finding that Hoxb1 expression in $\mathrm{r} 4$ depends on $\mathrm{Nkx} 6$ proteins reveals a regulatory interaction between HD proteins previously implicated in DV and AP patterning, and implies that Nkx6 proteins operate upstream of Hoxb1 to promote vMN and suppress $\mathrm{S}$ neurons in $\mathrm{r} 4$. Indeed, the reduction of Phox $2 \mathrm{~b}$ expression in progenitors (Gaufo et al. 2000) and the transformation of r4-derived MNs into an r2-like identity (Studer et al. 1996) observed in Hoxb1 mutants point towards an altered profile of vMN production in these mice. In $\mathrm{r} 4$ of Hoxb1 mutants, we observed a reduction and premature arrest of vMN generation that was associated with a complementary generation of ectopic $S$ neurons (Fig. 3a,b,d,e,j,k). Because the progenitor expression of Nkx6.1 and Nkx6.2 was unaffected by the loss of Hoxb1 (Fig. 3g,h), these findings favor the idea that a primary role for $\mathrm{Nkx} 6$ proteins in $\mathrm{AP}$ patterning is to sustain Hoxb1 expression in $\mathrm{r} 4$.

In Nkx6 mutants, Hoxb1 expression is gradually lost and a vMN-to-S neuron switch is observed. These data imply that Hoxb1 is required continuously throughout development in order for progenitors to retain their $\mathrm{r} 4$ identity. A prediction from such a hypothesis is that ventral r4-derived neurons generated prior to the loss of Hoxb1 in Nkx6 mutants should retain their r4 identity, whereas such neurons should be ablated in Hoxb1 mu- 
Pattyn et al.

Figure 2. Nkx6 proteins promote motor neuron generation and suppress S neurons in r4. (a) Graph showing the temporal profile of Phox $2 \mathrm{~b}$ expression in $\mathrm{Nkx} 2.2^{+}$progenitors in $\mathrm{r} 2, \mathrm{r} 4$, and $\mathrm{r} 5$ in wild-type (wt) and Nkx6 mutant mice. Note the reduced Phox $2 \mathrm{~b}$ expression in $\mathrm{r} 4$ of $N k x 6$ mutants. $(b-i) \mathrm{Mi}-$ crographs through $\mathrm{r} 4$ at E10.5 in controls $(b, d, f, h)$ and Nkx6 mutants $(c, e, g, i)$. The expression of Nkx2.2 $(d, e)$ and Nkx2.9 $(f, g)$ is similar in controls and Nkx6 mutants. The progenitor expression of Phox $2 \mathrm{~b}$ is reduced and primarily detected in dorsal $\mathrm{Nkx} 2.2^{+}$progenitors in $\mathrm{r} 4$ in Nkx6 mutants at E10.5 (i) compared to controls $(h)$. (j-o) Ectopic generation of $\mathrm{S}$ neurons in $\mathrm{r} 4$ of $N k x 6$ mutants. In $\mathrm{r} 4$ at E10.75, the reduction and ventral loss of Phox $2 b$ expression in Nkx6 mutants are accompanied by ectopic expression of pet1 $(j, k)$. At E11.5, 5HT expression is excluded in $\mathrm{r} 4$ of controls (1) but is detected ventral to Isl1 ${ }^{+}$vMNs in $N k x 6$ mutants $(m) .(n, o)$ Summary of vMN and S-cell generation in $\mathrm{r} 4$ of wild-type $\left(\mathrm{wt}^{\mathrm{t}}\right.$; $n)$ and Nkx6 mutant mice $(o) .(p-y)$ Nkx6 proteins are required to maintain $H o x b 1$ expression in $\mathrm{r} 4$. $(p-u)$ Micrographs showing expression of pet1 $(p, q)$, Hoxb1 $(r, s)$, and Hoxb2 $(t, u)$ in flat-mounted hindbrains at E11.5 in wild-type $\left(\mathrm{wt}_{;} p, r, t\right)$ and $N k x 6 \mathrm{mu}$ tants $(q, s, u)$. Note that the continuous expression of pet1 in the hindbrain in Nkx6 mutants $(q)$ is associated with a ventral loss of Hoxb1 expression in $\mathrm{r} 4 \mathrm{~s}$, arrowhead). $(v-y)$ Transverse sections showing Hoxb1 expression in $\mathrm{r} 4$. The expression of Hoxb1 is similar in controls $(v)$ and $N k x 6$ mutants $(w)$ at E9.5. A ventral down-regulation of Hoxb1 expression is observed in $\mathrm{r} 4$ at E10.5 in Nkx6 mutants $(y)$ compared to controls $(x)$. Brackets indicate $\mathrm{Nkx} 2.2^{+}$progenitor domain.

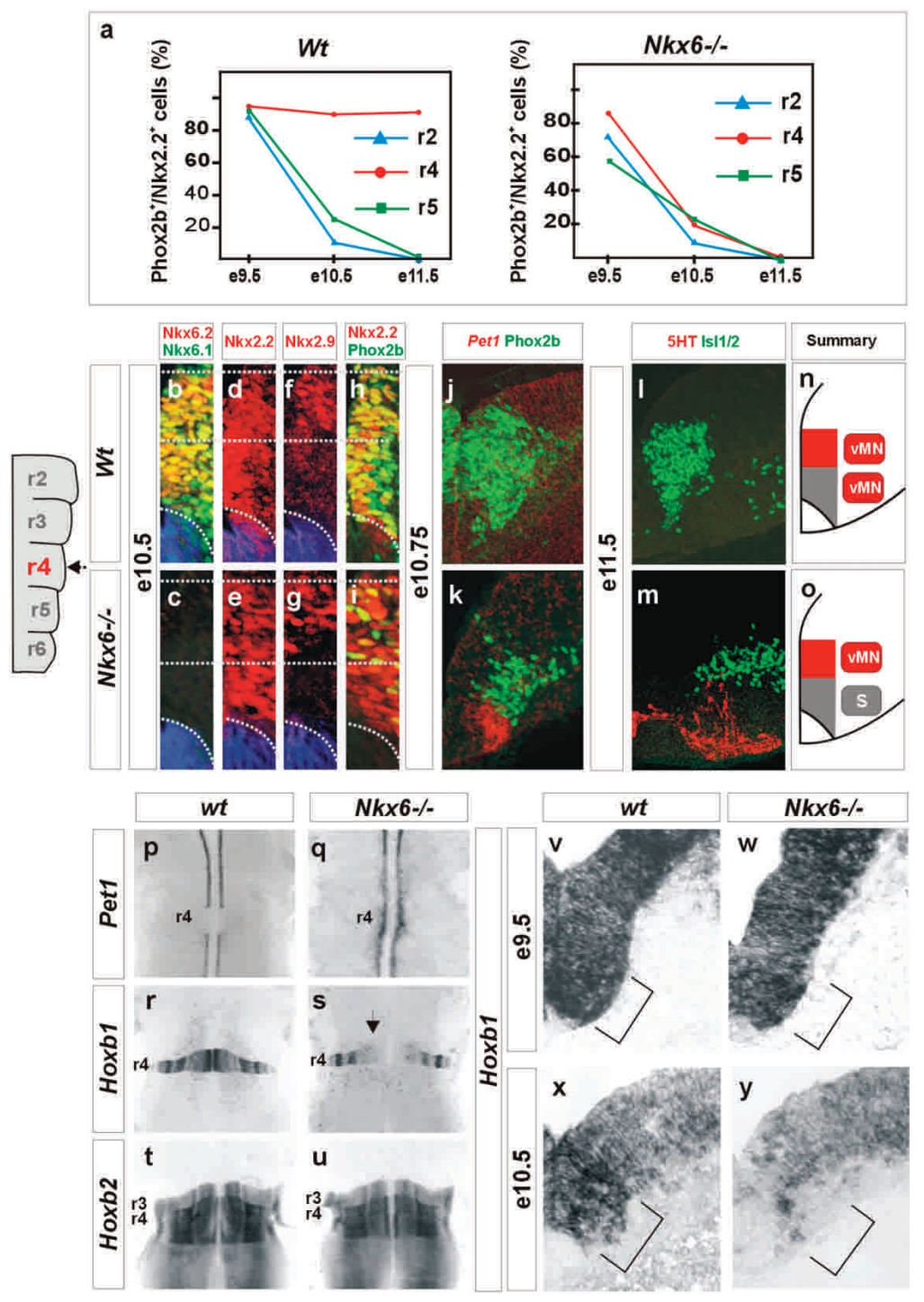

tants. In line with this idea, we found that Phox $2 \mathrm{~b}^{+} /$ $\mathrm{Gata}^{+}$inner ear efferent (iee) neurons (Karis et al. 2001), which are selectively generated from $\mathrm{Nkx} 2.2^{+}$progenitors in $\mathrm{r} 4$ prior to E10.5 (data not shown), are still detected in Nkx6 mutants (albeit in reduced numbers), but are completely missing in Hoxb1 mutants (Fig. 3q-s).

To examine further the control of vMN and $S$ neuron fate in $\mathrm{r} 4$, we also examined the patterns of neurogenesis in Hoxb2 mutants (Davenne et al. 1999), because Hoxb1 expression is down-regulated at a late stage in these mice (F. Rijli, unpubl.). In Hoxb2 mutants, Hoxb1 was expressed at high levels in the $\mathrm{Nkx} 2.2^{+}$domain in $\mathrm{r} 4$ at E10.5, and extensive down-regulation was not detected until E11.5 (Fig. 3m-p). Thus, a significant ventral loss of Hoxb1 expression occurs later in Hoxb2 mutants than in Nkx6 mutants (cf. Figs. 2y and 3o). The progenitor expression of Nkx6.1 and Nkx6.2 was unaffected in these mice (Fig. 3g,i). Strikingly, S neurons were also detected in $\mathrm{r} 4$ in Hoxb2 mutants, but the number of $\mathrm{S}$ neurons was considerably lower than that observed in both $N k x 6$ and Hoxb1 mutants (Fig. 3a-f; Fig. 2m,q). Moreover, the profile of vMN generation in Hoxb2 mutants appeared largely unaffected in $\mathrm{r} 4$ at E10.5 (Fig. 3j,1,q,t), and a significant reduction of Phox $2 \mathrm{~b}^{+} / \mathrm{Nkx} 2.2^{+}$vMN progenitors was not observed until E11.5 (data not shown). These data link the profile of vMN and S neuronal generation in $\mathrm{r} 4$ of Hoxb2 mutants to the temporal loss of Hoxb1 expression, rather than to the genetic ablation of Hoxb2, and they provide additional, albeit indirect, support for the idea that expression of Hoxb1 is necessary to promote vMN generation and to suppress $S$ neurons at this axial level.

We next turned our attention to the sequential production of vMNs and $S$ neurons observed at all axial levels, except in $r 4$. The finding that the down-regulation of Phox $2 \mathrm{~b}$ and Nkx2.9 in Nkx2.2 $2^{+}$progenitors anticipates the establishment of S-neuron progenitors (Fig. 1v) prompted us to characterize the loss of $\mathrm{S}$ neurons in Nkx2.2 mutant mice (Briscoe et al. 1999) in more detail. In Nkx2.2 mutants, the progenitor expression of Nkx6.1 

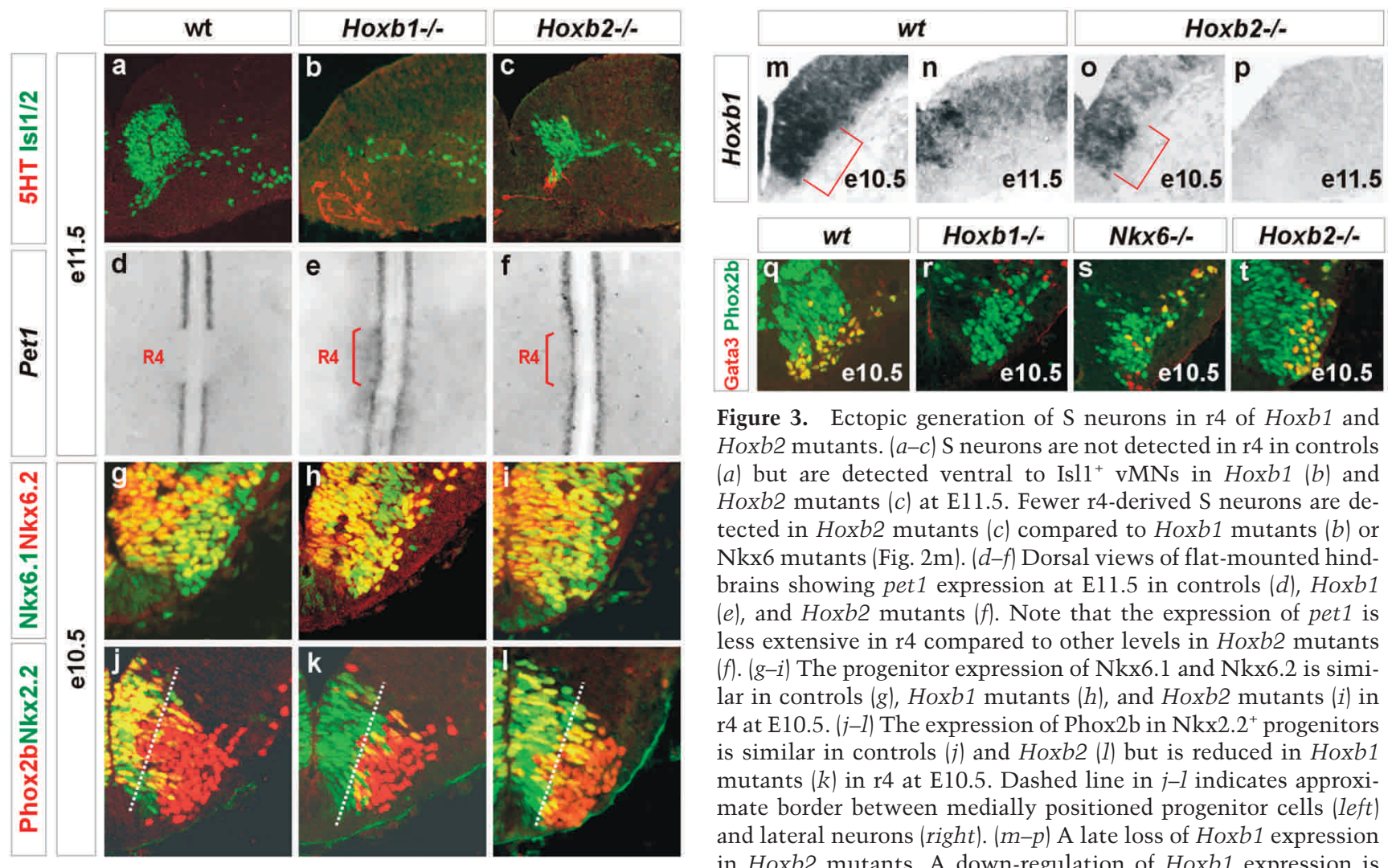

Figure 3. Ectopic generation of $\mathrm{S}$ neurons in $\mathrm{r} 4$ of $H o x b 1$ and Hoxb2 mutants. $(a-c)$ S neurons are not detected in $\mathrm{r} 4$ in controls (a) but are detected ventral to Isl1 ${ }^{+}$vMNs in Hoxb1 $(b)$ and Hoxb2 mutants $(c)$ at E11.5. Fewer r4-derived S neurons are detected in Hoxb2 mutants $(c)$ compared to Hoxb1 mutants $(b)$ or Nkx6 mutants (Fig. 2m). (d-f) Dorsal views of flat-mounted hindbrains showing pet 1 expression at E11.5 in controls $(d)$, Hoxb1 $(e)$, and Hoxb2 mutants $(f)$. Note that the expression of pet1 is less extensive in $\mathrm{r} 4$ compared to other levels in Hoxb2 mutants $(f) .(g-i)$ The progenitor expression of Nkx6.1 and Nkx6.2 is similar in controls $(g), H o x b 1$ mutants $(h)$, and Hoxb2 mutants (i) in r4 at E10.5. (j-1) The expression of Phox $2 \mathrm{~b}$ in Nkx2.2+ progenitors is similar in controls ( $j$ ) and Hoxb2 (1) but is reduced in Hoxb1 mutants $(k)$ in $r 4$ at E10.5. Dashed line in $j-l$ indicates approximate border between medially positioned progenitor cells (left) and lateral neurons (right). (m-p) A late loss of Hoxb1 expression in Hoxb2 mutants. A down-regulation of Hoxb1 expression is detected in $\mathrm{r} 4$ of Hoxb2 mutants $(o)$ compared to controls $(\mathrm{m})$, but the level of expression is still relatively high in the ventral Nkx2.2 ${ }^{+}$ domain (indicated by brackets in $m, o$ ) compared to $N k x 6$ mutants at the corresponding stage (cf. Fig. 2y). At E11.5, Hoxb1 is expressed in controls $(n)$ but is essentially extinguished in $\mathrm{r} 4$ of Hoxb2 mutants $(p)$. ( $q-t)$ Generation of iee neurons in r4 of wild-type (wt), Hoxb2, Nkx6, and Hoxb1 mutants: iee neurons that coexpress Phox2b and GATA3 (yellow) can be detected at r4 levels at E10.5 in controls $(q), H o x b 2(t)$, and Nkx6 mutants $(s)$, but these neurons are completely missing in Hoxb1mutants $(r)$.

and Nkx6.2 was unaffected and the expression pattern of Nkx2.9 and Phox2b was similar to controls at early stages (E9.5; data not shown). Remarkably, the subsequent dorsal restriction of Nkx2.9 and Phox $2 b$ expression observed in controls failed to occur in Nkx2.2 mutants (Fig. 4a-h). The loss of $\mathrm{S}$ neurons was further accompanied by a total increase in vMN numbers $(33 \% \pm 6 \%$ S.D., $\mathrm{n}=4)$ in $\mathrm{r} 5$ at E11, and many vMNs occupied a position at which $S$ neurons are normally detected (Fig. 4i-n; data not shown). These data reveal that Nkx2.2 is required for the temporal conversion of vMN progenitors into S-neuron progenitors. Nkx2.9 cannot compensate the loss of Nkx2.2 function, despite a ventral expansion of Nkx2.9 expression (Fig. 4e,f) at stages when $S$ neurons are normally being specified. Thus, although Nkx2.2 and Nkx2.9 have redundant functions in other aspects of neural patterning (Briscoe et al. 1999, 2000), our analysis reveals a novel role for Nkx2.2 in the establishment of S-neuron progenitors.

In normal conditions, as well as in Nkx2.2, Nkx6, Hoxb1, and Hoxb2 mutant mice, there is a strict correlation between the progenitor expression of Phox $2 \mathrm{~b}$ and the selection of vMN fate. This observation suggests that Phox $2 b$ may be a key mediator of the switch that determines whether $\mathrm{Nkx} 2.2^{+}$progenitors will select a $\mathrm{vMN}$ or
$S$ fate. To examine this possibility, we analyzed Phox $2 b$ mutant mice (Pattyn et al. 2000). All vMNs are missing in Phox $2 b$ mutants, and many progenitors are arrested in an $\mathrm{Nkx} 2.2^{+}$state, most likely reflecting the role of Phox $2 \mathrm{~b}$ to induce pro-neural bHLH proteins in the vMN pathway (Dubreuil et al. 2000; Pattyn et al. 2000). Importantly, not all cells fail to exit the cell cycle (Pattyn et al. 2000), and this allowed us to determine the identity of the neurons derived from Nkx2.2+ progenitors in $P$ hox $2 b$ mutants. Strikingly, the loss of vMNs observed in these mice was accompanied by premature expression of pet 1 and S-neuron generation at all axial levels of the hindbrain at E10.5, including r4 (Fig. 5a-d; data not shown). The production of ectopic $S$ neurons was extensive in $\mathrm{r} 4$ at E11.5 (Fig. 5e,f), despite the fact that the progenitor expression of $\mathrm{Nkx} 2.2, \mathrm{Nkx} 6.1$, and $\mathrm{Nkx} 6.2$ at all axial levels, and Hoxb1 in r4, appeared unaffected (Fig. 5g,h; data not shown). Moreover, cells that coexpressed serotonin $(5 \mathrm{HT})$ and LacZ driven by the Phox $2 b$ locus (Pattyn et al. 2000) could be detected (Fig. 5e,f), providing direct evidence that vMN progenitors, in the absence of Phox2b, give rise to $S$ neurons. These findings establish a requirement for Phox $2 b$ to suppress $S$-neuronal fate in vMN progenitors, and predict that the progressive extinction of Phox $2 \mathrm{~b}$ in Nkx2.2+ progenitors is necessary 
Pattyn et al.

Figure 4. Nkx2.2 is required for the temporal establishment of S-neuron progenitors. $(a-j)$ Transverse sections through the ventral hindbrain at the $\mathrm{r} 5$ level in wildtype (wt) and Nkx2.2 mutant mice. The progenitor expression of Nkx6.1 and Nkx6.2 is similar in control embryos $(a, c)$ and in $N k x 2.2$ mutants $(b, d)$ at E10.5. The dorsal restriction of Nkx2.9 and Phox $2 \mathrm{~b}$ observed in control embryos $(e, g)$ fails to occur in Nkx2.2 mutants $(f, h)$, and the expression of these proteins extends ventrally to the dorsal boundary of Shh expression in the floor plate $(a-h$, blue staining). In $r 5$ at E11, Pet1 and 5HT expression is missing, and vMNs identified by Phox $2 \mathrm{~b}$ and Isl expression are increased in number and ventrally expanded (indicated by dashed line) in Nkx2.2 mutants (i-1). $(m, n)$ Summary of vMN and S-cell generation in r5 in controls $(m)$ and $N k x 2.2 \mathrm{mu}-$ tants $(n)$.

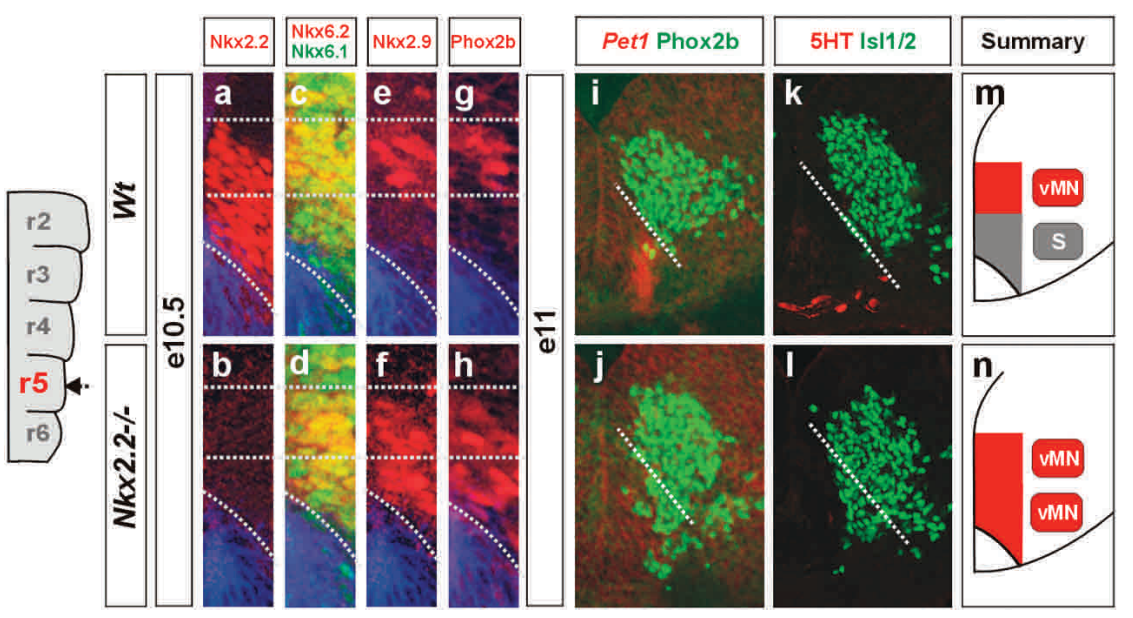

for the generation of $S$ neurons. Indeed, the suppression of $\mathrm{S}$-neuronal fate in $\mathrm{r} 4$ provides direct support for this idea, because the extended production of motor neurons at the expense of $\mathrm{S}$ neurons at this level depends on the prolonged activation of Phox $2 \mathrm{~b}$ in all Nkx $2.2^{+}$progenitors.

\section{Discussion}

In this study, we examined the sequential generation of vMNs and $\mathrm{S}$ neurons from a common pool of Nkx2.2 progenitor cells in the developing mouse hindbrain. We obtained evidence that $\mathrm{Nkx} 2.2^{+}$progenitors undergo changes in progenitor cell identity over time, and that these changes reflect a conversion of vMN progenitors into S-neuron progenitors. Nkx2.2 is required in this process, and the loss of $\mathrm{S}$ neurons in Nkx2.2 mutants (Briscoe et al. 1999) is associated with an extended production of vMNs and a failure to suppress the progenitor expression of Phox $2 b$ and Nkx2.9. How the switch from vMN to S-neuron generation is initiated is still unclear, but the progressive dorsal restriction in Phox $2 \mathrm{~b}$ and Nkx2.9 expression indicates that a signal provided by the floor plate may be involved. Because the pattern of Nkx2.2 expression is unchanged over time, a key activity of such a signal would be predicted to induce, or activate, a cofactor necessary for Nkx2.2 to convert $\mathrm{Nkx} 2.2^{+} / \mathrm{Nkx} 2.9^{+} / \mathrm{Phox} \mathrm{b}^{+}$vMN progenitors into Nkx2.2 $2^{+}$Nkx2.9 $9^{-}$Phox $2 \mathrm{~b}^{-} \mathrm{S}$ neuron progenitors (Fig. 5i).

Our analysis further reveals a close regulatory link between Nkx6 proteins and Hoxb1 in $\mathrm{r} 4$ that directly influences the spatial and temporal control of neuronal cell fate. In both Nkx6 and Hoxb1 mutant mice, S neurons are generated in $\mathrm{r} 4$ and the production of vMNs is impaired. We provide evidence that the AP-specific role for Nkx6.1 and Nkx6.2 is indirect and reflects a requirement for these proteins to sustain Hoxb1 expression. Hoxb1, in turn, promotes vMN generation and suppresses $\mathrm{S}$ neurons by extending the spatial and temporal activation of Phox $2 \mathrm{~b}$ expression in Nkx $2.2^{+}$progenitors. In this respect, it is possible that Hoxb1 directly activates Phox $2 b$ expression and in this way simply overrules the establishment of S-neuron progenitors evident at other axial levels of the hindbrain (Fig. 5i).

The down-regulation of Hoxb1 expression in Nkx6 and Hoxb2 mutants also provides insight into the temporal requirement for Hoxb1 to confer r4-positional identity in the hindbrain. The initial phase of Hoxb1 expression is unaffected in $N k \times 6$ mutants, and a significant ventral loss of Hoxb1 expression is detected first at approximately E10.5. In this situation, and in contrast to Hoxb1 mutants (Studer et al. 1996; Gaufo et al. 2000), vMNs generated prior to E10.5 retain $\mathrm{r} 4$ characteristics, whereas late-born vMNs are missing because $\mathrm{r} 4$ progenitors instead adopt an S-neuronal fate. Moreover, we find that the even later loss of Hoxb1 expression observed in Hoxb2 mutants correlates with a mild phenotype in which only a few $\mathrm{r} 4$ progenitors adopt an S-neuronal fate. In addition to an early requirement to establish $\mathrm{r} 4$ identity (Studer et al. 1996; Gaufo et al. 2000), these observations indicate that the expression of Hoxb1 must be maintained over time in order for progenitors to retain their r4-positional identity and select a fate appropriate to their developmental history.

The sequential production of distinct classes of neurons represents a central strategy to establish cell diversity in the developing CNS, but few molecular determinants involved in this process have been identified (Livesey and Cepko 2001; Monuki and Walsh 2001). Our data establish that the sequential production of vMNs and S neurons in the hindbrain critically depends on HD proteins previously implicated in DV and AP patterning, assigning new roles for these proteins in the temporal 


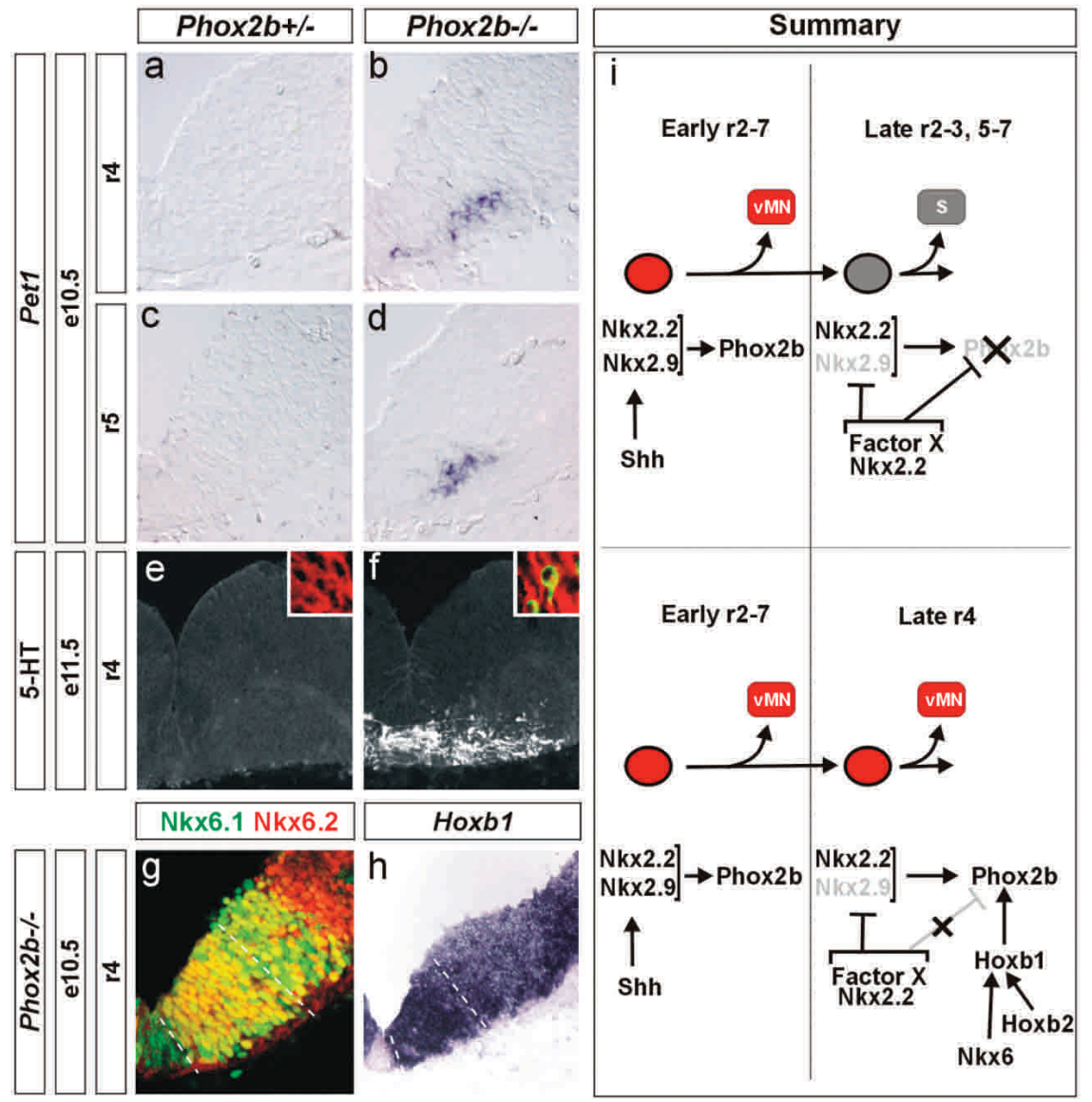

Figure 5. Phox $2 \mathrm{~b}$ is required to suppress the premature generation of $S$ neurons in the hindbrain. $(a-f)$ Pet1 is prematurely and ectopically expressed in Phox2b mutants. No pet 1 expression is detected in $\mathrm{r} 4$ or r5 of Phox $2 b^{+/-}$control embryos at E10.5 $(a, c)$, whereas a premature and ectopic expression of pet 1 was detected at this stage in Phox $2 b^{-l-}$ embryos $(b, d)$. $5 \mathrm{HT}$ expression could also be detected at these levels at E10.5 (data not shown). 5HT is not detected in $\mathrm{r} 4$ in controls $(e)$ but is extensively expressed at this level in Phox $2 b^{-/-}$embryos at E11.5 $(f)$. Inserted micrographs in $e$ and $f$ show LacZ expression under control of the Phox $2 b$ locus (red) and the expression of 5HT (green). $(g, h)$ The progenitor expression of Nkx6.1 and Nkx6.2 $(\mathrm{g})$ and Hoxb1 $(h)$ is unaffected in $\mathrm{r} 4$ of Phox $2 b^{-/-}$embryos at E10.5. (i) Model of vMN and S-neuron generation in the hindbrain. At early stages at all axial levels (r2-r7), Shh signaling induces vMN progenitors that express $\mathrm{Nkx} 2.2, \mathrm{Nkx} 2.9$, Nkx6.1, Nkx6.2, and Phox2b. The expression of Phox $2 \mathrm{~b}$ promotes vMNs and suppresses $\mathrm{S}$ neurons. At later stages (at all levels except r4), Nkx2.9 and Phox $2 \mathrm{~b}$ are suppressed in ventral $\mathrm{Nkx} 2.2^{+}$progenitors, converting vMN progenitors into Sneuron progenitors. In the absence of Phox $2 b$, cells select the S-neuronal fate. The establishment of S-neuron progenitors may be mediated by a signal produced by the floor plate, that induces or activates a factor (Factor $\mathrm{X}$ ) that is necessary for Nkx2.2 to suppress Nkx2.9 and Phox $2 \mathrm{~b}$ expression. In $\mathrm{r} 4$, all Nkx2.2 progenitors produce vMNs also at late stages, and the generation of $\mathrm{S}$ neurons is blocked. At this level, Hoxb1 ensures that all Nkx2.2+ progenitors express Phox2b. The sustained expression of Hoxb1 in $\mathrm{r} 4$, in turn, depends on Nkx6 and Hoxb2 proteins. Factor $\mathrm{X}$ is predicted to be induced also in $\mathrm{r} 4$ (because $\mathrm{Nkx} 2.9$ is suppressed and $\mathrm{S}$ neurons are generated if Hoxb1 is missing or is down-regulated). Hoxb1 must therefore override the establishment of S-neuron progenitors evident at other levels, possibly by directly activating Phox $2 \mathrm{~b}$ expression in all Nkx2.2 progenitors. For further details, see text.

control of neuronal fate determination. We show that the primary role for Nkx and Hox HD proteins is to coordinate the temporal and spatial expression of Phox $2 b$ in neural progenitors, and that Phox $2 b$ in turn acts as a molecular switch that determines whether progenitors select a vMN or S-neuronal fate. The role of Phox $2 b$ to promote early-born neurons and suppress lateborn neurons shows a high degree of similarity to the temporal determinant Hunchback in the Drosophila CNS (Isshiki et al. 2001). In Drosophila, a cell cycledependent clock mechanism has been proposed to underlie the regulation of temporal determinants (Isshiki et al. 2001). The variable generation of vMN and S neurons along the AP axis implies that the temporal control of Phox $2 \mathrm{~b}$ expression in the hindbrain is uncoupled from the cell cycle and, as discussed above, appears instead to rely on the integrated activity of Nkx and Hox proteins. Recent studies in the spinal cord have implicated that the switch from generating somatic MNs to produce oligodendrocytes may be triggered by an expansion of $\mathrm{Nkx} 2.2$ expression into the neighboring Olig2 ${ }^{+}$domain
(Zhou et al. 2001). However, the precise role of these proteins in this switch remains unclear, because a neuron-to-glial switch is still observed in both Nkx2.2 (Qi et al. 2001) and Olig2 (Lu et al. 2002; Zhou and Anderson 2002) mutant mice. Nevertheless, data begin to suggest that determinants that control spatial patterning generally may be associated with temporal aspects of neural fate determination. In this view, the sequential control of neuronal fate specification would be mechanistically analogous to spatial patterning, but with the notion that the expression pattern of intrinsic determinants is dynamic and modulated over time.

\section{Materials and methods}

\section{Mouse strains}

The generation and genotyping of mouse mutants have been reported: Nkx6.1 (Sander et al. 2000), Nkx6.2 (Vallstedt et al. 2001), Hoxb1 (Studer et al. 1996), Hoxb2 (Davenne et al. 1999), Nkx2.2 (Briscoe et al. 1999), and Phox2b (Pattyn et al. 2000). 
Immunohistochemistry and in situ hybridization histochemistry

Immunohistochemical localization of proteins was performed as described (Briscoe et al. 2000) using the following antibodies: mouse $(\mathrm{m})$, rabbit $(\mathrm{r})$, and guinea pig (gp) Is11/2, gp Nkx2.9 (Briscoe et al. 2000), gp Nkx6.2 (Vallstedt et al. 2001), m Gata3 (Santa Cruz Biotechnology), $\mathrm{m}$ and $\mathrm{r}$ Nkx2.2 (Ericson et al. 1997), r Phox2b (Pattyn et al. 2000), and r Nkx6.1 (Briscoe et al. 1999). Serotonergic (S) neurons were detected by r Serotonin (5HT) antibody (Sigma). In situ hybridization histochemistry on sections or as whole mounts were performed as described (Wilkinson 1992; Schaeren-Wiemers and Gerfin-Moser 1993) using pet1, Hoxb1, Hoxb2, Is11, Hoxb4, Hoxa1, and Hoxa2 probes.

\section{BrdU labeling}

$\mathrm{BrdU}$ (Sigma) was injected intraperitoneally into pregnant mice $(0.1 \mathrm{mg} / \mathrm{g}$ of body weight) at E8.5, E9.5, E10, E10.5, E11, and E11.5. Embryos were harvested at E12.5 and analyzed for incorporation of $\mathrm{BrdU}$ in motor neurons and $\mathrm{S}$ neurons using BrdU antibodies in combinations with Phox $2 \mathrm{~b}$, Isl1/2, 5HT, Gata3 antibodies.

\section{Acknowledgments}

We thank A. Gavalas, N. Grillet, M. Sander, and L. Sussel for mice; T. Jessell, S. Arber, and B. Novitch for reagents; J. Briscoe, P. Flodby, J. Frisén, C. Goridis, T. Jessell, B. Novitch, T. Perlmann, and M. Sander for helpful discussions and comments on the manuscript. A.P. was supported by a postdoctoral fellowship from the Karolinska Institute (KI). J.M.D is supported by the GABBA program of the University of Porto and the Portuguese Foundation for Science and Technology. O.A.S. was supported by a fellowship from La Ligue Nationale contre le Cancer. F.M.R. is supported by grants from the EEC Quality of Life Program (\#QLG2-CT01-01467), the ARC, the Ministère pour le Recherche and by institutional funds from CNRS, INSERM, and Hôpital Universitaire de Strasbourg. J.E. is supported by the Royal Swedish Academy of Sciences by a donation from the Wallenberg Foundation, The Swedish Foundation for Strategic Research, The Swedish National Research Council, Project A.L.S., the KI, and by the EC network grant, Brainstem Genetics, QLRT-2000-01467. This study is dedicated to C.Z.

The publication costs of this article were defrayed in part by payment of page charges. This article must therefore be hereby marked "advertisement" in accordance with 18 USC section 1734 solely to indicate this fact.

\section{References}

Aitken, A.R. and Tork, I. 1988. Early development of serotonincontaining neurons and pathways as seen in wholemount preparations of the fetal rat brain. J. Comp. Neurol. 274: 3247.

Bell, E., Wingate, R.J., and Lumsden, A. 1999. Homeotic transformation of rhombomere identity after localized Hoxb1 misexpression. Science 284: 2168-2171.

Briscoe, J., Sussel, L., Serup, P., Hartigan-O'Connor, D., Jessell, T. M., Rubenstein, J. L., and Ericson, J.1999. Homeobox gene Nkx2.2 and specification of neuronal identity by graded Sonic hedgehog signalling. Nature 398: 622-627.

Briscoe, J., Pierani, A., Jessell, T.M., and Ericson, J. 2000. A homeodomain code specifies progenitor cell identity and neuronal fate in the ventral neural tube. Cell 101: 435-445.

Davenne, M., Maconochie, M.K., Neun, R., Pattyn A., Chambon, P., Krumlauf, R., and Rijli, F.M. 1999. Hoxa2 and Hoxb2 control dorsoventral patterns of neuronal development in the rostral hindbrain. Neuron 22: 677-691.

Dubreuil, V., Hirsch, M.R., Pattyn, A., Brunet, J-F., and Goridis, C. 2000. The Phox $2 \mathrm{~b}$ transcription factor coordinately regulates neuronal cell cycle exit and identity. Development 127: 5191-5201.

Ericson J., Rashbass P., Schedl A., Brenner-Morton S., Kawakami A., van Heyningen V., Jessell T.M., Briscoe J. 1997. Pax6 controls progenitor cell identity and neuronal fate in response to graded Shh signaling. Cell 90: 169-180.

Gaufo, G.O., Flodby, P., and Capecchi, M.R. 2000. Hoxb1 controls effectors of sonic hedgehog and Mash1 signaling pathways. Development 127: 5343-5354.

Guthrie, S., Muchamore, I., Kuroiwa, A., Marshall, H., Krumlauf, R., and Lumsden, A. 1992. Neuroectodermal autonomy of Hox-2.9 expression revealed by rhombomere transpositions. Nature 356: 157-159.

Hendricks, T., Francis, N., Fyodorov, D., and Deneris, E.S. 1999. The ETS domain factor Pet- 1 is an early and precise marker of central serotonin neurons and interacts with a conserved element in serotonergic genes. J. Neurosci. 19: 10348-10356.

Isshiki, T., Pearson, B., Holbrook, S., and Doe, C.Q. 2001. Drosophila neuroblasts sequentially express transcription factors which specify the temporal identity of their neuronal progeny. Cell 106: 511-521.

Jessell, T.M. 2000. Neuronal specification in the spinal cord: Inductive signals and transcriptional codes. Nat. Rev. Genet. 1: 20-29.

Karis, A., Pata I., van Doorninck, J.H., Grosveld, F., de Zeeuw, C.I., de Caprona, D., and Fritzsch, B. 2001. Transcription factor GATA-3 alters pathway selection of olivocochlear neurons and affects morphogenesis of the ear. I. Comp. Neurol. 429: 615-630.

Leber, S.M. and Sanes, J.R. 1995. Migratory paths of neurons and glia in the embryonic chick spinal cord. J Neurosci. 15: 1236 1248.

Lidov, H.G. and Molliver, M.E. 1982. Immunohistochemical study of the development of serotonergic neurons in the rat CNS. Brain Res. Bull. 9: 559-604.

Livesey, F.J. and Cepko, C.L. 2001. Vertebrate neural cell-fate determination: Lessons from the retina. Nat. Rev. Neurosci. 2: 109-118.

Lu, Q.R., Sun, T., Zhu, Z., Ma, N., Garcia, M., Stiles, C.D., and Rowitch, D.H. 2002. Common developmental requirement for Olig function indicates a motor neuron/oligodendrocyte connection. Cell 109: 75-86.

Lumsden, A. and Krumlauf, R. Patterning the vertebrate neuraxis. 1996. Science 274: 1109-1115.

Monuki, E.S. and Walsh, C.A. 2001. Mechanisms of cerebral cortical patterning in mice and humans. Nat. Neurosci. 4: 1199-1206.

Pattyn, A., Hirsch, M., Goridis, C., and Brunet, J-F. 2000. Control of hindbrain motor neuron differentiation by the homeobox gene Phox2b. Development 127: 1349-1358.

Qi, Y., Cai, J., Wu, Y., Wu, R., Lee, J., Fu, H., Rao, M., Sussel, L., Rubenstein, J., and Qiu, M. 2001. Control of oligodendrocyte differentiation by the Nkx2.2 homeodomain transcription factor. Development 128: 2723-2733.

Sander, M., Paydar, S., Ericson, J., Briscoe, J., Berber, E., German, M., Jessell, T.M., and Rubenstein, J.L. 2000. Ventral neural patterning by Nkx homeobox genes: Nkx6.1 controls somatic motor neuron and ventral interneuron fates. Genes \& Dev. 14: 2134-2139. 
Schaeren-Wiemers, N. and Gerfin-Moser, A. 1993. A single protocol to detect transcripts of various types and expression levels in neural tissue and cultured cells: In situ hybridization using digoxigenin-labeled cRNA probes. Histochemistry 100: 431-440.

Simon, H., Hornbruch, A., and Lumsden, A. 1995. Independent assignment of antero-posterior and dorso-ventral positional values in the developing chick hindbrain. Curr. Biol. 5: 205214.

Studer, M., Lumsden, A., Ariza-McNaughton, L., Bradley, A., and Krumlauf, R. 1996. Altered segmental identity and abnormal migration of motor neurons in mice lacking Hoxb-1. Nature 384: 630-634.

Taber-Pierce, E. 1973. Time of origin of neurons in the brainstem of the mouse. Prog. Brain Res. 40: 53-65.

Vallstedt, A., Muhr, J., Pattyn, A., Pierani, A., Mendelsohn, M., Sander, M., Jessell, T.M., and Ericson, J. 2001. Different levels of repressor activity assign redundant and specific roles to Nkx6 genes in motor neuron and interneuron specification. Neuron 31: 743-755.

Wilkinson, D.G. 1992. Whole-mount in situ hybridization of vertebrate embryos. In: In situ hybridization. A practical approach (ed. D.G. Wilkinson), p. 75. IRL Press, Oxford, UK.

Yun, K., Fishman, S., Johnson, J., Hrabe de Angelis, M., Weinmaster, G., and Rubenstein, J.L.R. 2002. Modulation of the notch signalling by Mash 1 and $\mathrm{Dlx} 1 / 2$ regulates sequential specification and differentiation of progenitor cell types in the subcortical telencephalon. Development 129:50295040.

Zhou, Q. and Anderson, D.J. 2002. The bHLH transcription factors OLIG2 and OLIG1 couple neuronal and glial subtype specification. Cell 109: 61-73.

Zhou, Q., Choi, G., and Anderson, D.J. 2001. The bHLH transcription factor Olig2 promotes oligodendrocyte differentiation in collaboration with Nkx2.2. Neuron 31: 791-807. 


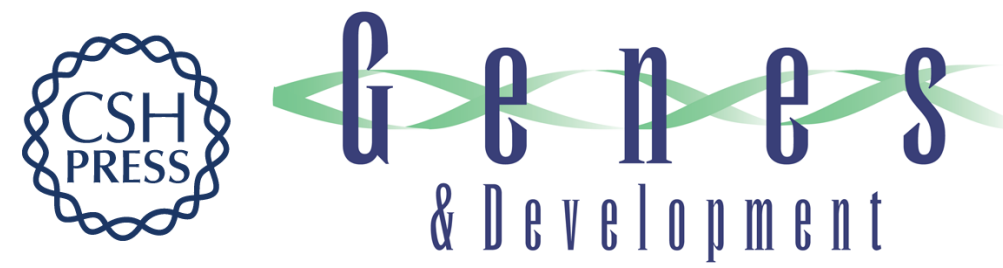

\section{Coordinated temporal and spatial control of motor neuron and serotonergic neuron generation from a common pool of CNS progenitors}

Alexandre Pattyn, Anna Vallstedt, José M. Dias, et al.

Genes Dev. 2003, 17:

Access the most recent version at doi:10.1101/gad.255803

References This article cites 28 articles, 9 of which can be accessed free at: http://genesdev.cshlp.org/content/17/6/729.full.html\#ref-list-1

License

Email Alerting

Service

Receive free email alerts when new articles cite this article - sign up in the box at the top right corner of the article or click here.

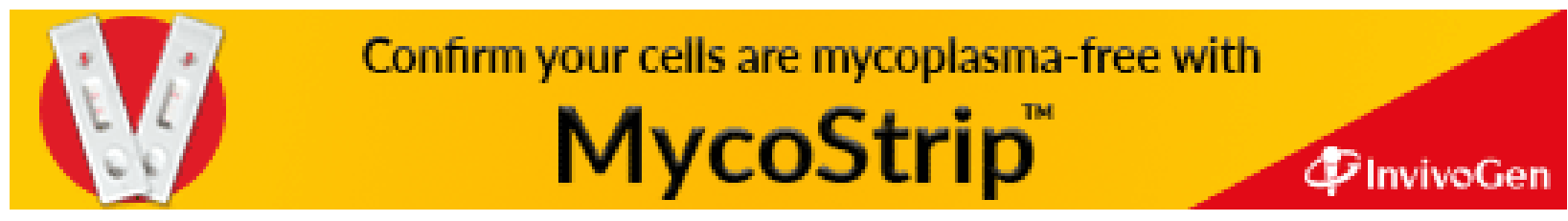

